PROCEEDINGS OF THE

AMERICAN MATHEMATICAL SOCIETY

Volume 140, Number 2, February 2012, Pages 607-614

S 0002-9939(2011)10922-5

Article electronically published on May 12, 2011

\title{
A NEW ASYMPTOTIC BEHAVIOR OF SOLUTIONS TO THE CAMASSA-HOLM EQUATION
}

\author{
LIDIAO NI AND YONG ZHOU \\ (Communicated by Walter Craig)
}

\begin{abstract}
The present work is mainly concerned with an algebraic decay rate of the strong solution to the Camassa-Holm equation in $L^{\infty}$-space. In particular, it is proved that the solution decays algebraically with the same exponent as that of the initial datum.
\end{abstract}

\section{INTRODUCTION AND MAIN RESULTS}

In this paper, we consider the Camassa-Holm equation

$$
u_{t}-u_{x x t}+3 u u_{x}=2 u_{x} u_{x x}+u u_{x x x}, x \in \mathbb{R}, t>0,
$$

which models wave motion in shallow water, with $u(x, t)$ representing the height of the water above a flat bottom at time $t$ in the spatial $x$ direction. This shallow water equation appears in the context of hereditary symmetries studied by Fuchssteiner and Fokas [6] as a bi-Hamiltonian generalization of KdV. It was first written explicitly and derived physically by approximating directly the Hamiltonian for Euler's equations in the shallow water regime [4].

Some satisfactory results have recently been obtained for equation (1.1). Local well-posedness for the initial datum $u_{0}(x) \in H^{s}$ with $s>3 / 2$ was proved by several authors; see for example [5, 11]. For the initial data with lower regularity, we refer to the recent paper 1, 12. The long-time behavior of solutions has been studied. Moreover, conditions which guarantee their global existence and wave breaking for a large class of initial data has been established in [3, 7, 13, 14. A new proof and analysis for blow-up profile was given recently in [9].

If one sets $Q=\left(1-\partial_{x}^{2}\right)$, then the operator $Q^{-1}$ can be expressed by

$$
Q^{-1} f=G * f=\frac{1}{2} \int_{\mathbb{R}} e^{-|x-y|} f(y) d y,
$$

Received by the editors April 21, 2010 and, in revised form, November 28, 2010.

2010 Mathematics Subject Classification. Primary 37L05; Secondary 35Q58, 26A12.

Key words and phrases. Camassa-Holm equation, asymptotic behavior.

The second author is the corresponding author and is partially supported by the Zhejiang Innovation Project (Grant No. T200905), ZJNSF (Grant No. R6090109) and NSFC (Grant No. 10971197).

(C)2011 American Mathematical Society Reverts to public domain 28 years from publication 
for all $f \in L^{2}(\mathbb{R})$ with $G(x)=\frac{1}{2} e^{-|x|}$. Using this identity, it is convenient to rewrite the equation (1.1) as a quasi-linear equation of hyperbolic type:

$$
u_{t}+u u_{x}+\partial_{x} G *\left(u^{2}+\frac{1}{2} u_{x}^{2}\right)=0, x \in \mathbb{R}, t>0 .
$$

In a recent work [2], it was shown that $u$ cannot preserve compact support in a non-trivial time interval except $u \equiv 0$. Later, in $[8$, Himonas, Misiolek, Ponce and Zhou give a detailed description of the corresponding solution $u(x, t)$ with $u_{0}(x)=$ $u(x, t=0) \in C_{0}^{\infty}(\mathbb{R})$, which implies infinite propagation speed for the CamassaHolm equation. Furthermore, it is proved that if $\left|u_{0}(x)\right|,\left|u_{0 x}(x)\right| \sim O\left(e^{-\theta x}\right)$, as $|x| \rightarrow \infty$, then $|u(x, t)|,\left|u_{x}(x, t)\right| \sim O\left(e^{-\theta x}\right)$, as $|x| \rightarrow \infty$ uniformly in the time interval $[0, T]$. Here $\theta \in(0,1)$ and $T$ is in the lifespan. Another important result is that if for any $\alpha \in(1 / 2,1),\left|u_{0}(x)\right| \sim O\left(e^{-x}\right)$ and $\left|u_{0 x}(x)\right| \sim O\left(e^{-\alpha x}\right)$ hold true, then $|u(x, t)| \sim O\left(e^{-x}\right)$ uniformly in the time interval $[0, T]$.

Here we introduce the notation

$$
|f(x)| \sim O(|g(x)|), \quad \text { as } \quad|x| \rightarrow \infty \quad \text { if } \quad \lim _{x \rightarrow \infty} \frac{|f(x)|}{|g(x)|}=L,
$$

where $L$ is a positive constant.

However, exponential decay is too fast; a natural question is how to change the situation for slower decaying initial datum. More precisely, we want to know the decay rate of $u(x, t)$ if $u_{0}(x)$ is just algebraically decreasing as $|x| \rightarrow \infty$. Fortunately, we have the following answers.

Theorem 1.1. Assume that for some $T>0$ and $s>3 / 2, u \in C\left([0, T] ; H^{s}(\mathbb{R})\right)$ is a strong solution of the initial value problem associated to equation (1.2) and that $u_{0}(x)=u(x, 0)$ satisfies

$$
\left|u_{0}(x)\right|, \quad\left|u_{0 x}(x)\right| \sim O\left((1+x)^{-\alpha}\right), \quad \text { as } \quad|x| \rightarrow \infty,
$$

for any $\alpha>1 / 2$. Then

$$
|u(x, t)|, \quad\left|u_{x}(x, t)\right| \sim O\left((1+x)^{-\alpha}\right), \quad \text { as } \quad|x| \rightarrow \infty
$$

uniformly in the time interval $[0, T]$.

Theorem 1.2. Assume that for some $T>0$ and $s>3 / 2, u \in C\left([0, T] ; H^{s}(\mathbb{R})\right)$ is a strong solution of the initial value problem associated to equation (1.2) and that $u_{0}(x)=u(x, 0)$ satisfies

$$
\left|u_{0}(x)\right| \sim O\left((1+x)^{-\alpha}\right), \quad\left|u_{0 x}(x)\right| \sim O\left((1+x)^{-\beta}\right), \quad \text { as } \quad|x| \rightarrow \infty,
$$

for any $\alpha>1 / 2$ and $\beta \in(\alpha / 2, \alpha)$. Then

$$
|u(x, t)| \sim O\left((1+x)^{-\alpha}\right), \quad \text { as } \quad|x| \rightarrow \infty
$$

uniformly in the time interval $[0, T]$.

Remark 1.3. Similar results can be established for the Holm-Staley $b$-family of equations and $\theta$-equations, which give supplements for [15] and [10]. 


\section{An ELEMENTARY LEMma}

Mainly, we follow the strategy in [8], but with different construction. For any $N \in \mathbb{Z}^{+}$, we define the cut-off function $\varphi_{N}(x)$ as follows:

$$
\varphi_{N}(x)= \begin{cases}1, & x \leq 0, \\ (1+x)^{\alpha}, & x \in(0, N), \\ (1+N)^{\alpha}, & x \geq N .\end{cases}
$$

A simple calculation shows that for all $N \in \mathbb{Z}^{+}$, one has

$$
0 \leq \varphi_{N}^{\prime}(x) \leq \varphi_{N}(x), \quad \text { a.e. } \quad x \in \mathbb{R} .
$$

The main goal of this section is to give a rough estimate for $\left|\varphi_{N} \partial_{x} G * f^{2}\right|$ for any appropriate function $f$, based on delicate $L^{\infty}$-estimates. The following elementary lemma will play an important role in Section 3.

Lemma 2.1. For a given function $\varphi_{N}(x)$ in (2.1), there exists a constant $C_{\alpha}>0$ which depends only on $\alpha$, such that for any positive integer $N$

$$
\left|\varphi_{N} \partial_{x} G * f^{2}\right| \leq C_{\alpha}\left\|\varphi_{N} f\right\|_{L^{\infty}}\|f\|_{L^{\infty}},
$$

for any $N \in \mathbb{Z}^{+}$and $f \in L^{1}(\mathbb{R}) \cap L^{\infty}(\mathbb{R})$.

Remark 2.2. Since $\partial_{x}^{2} G=G-\delta$, the argument in (2.3) also shows that

$$
\left|\varphi_{N} \partial_{x}^{2} G * f^{2}\right| \leq C_{\alpha}\left\|\varphi_{N} f\right\|_{L^{\infty}}\|f\|_{L^{\infty}} .
$$

Proof. First we should show that there exists $C_{\alpha}>0$, depending only on $\alpha>1 / 2$, such that for any $N \in \mathbb{Z}^{+}$,

$$
\varphi_{N}(x) \int_{-\infty}^{\infty} e^{-|x-y|} \frac{1}{\varphi_{N}(y)} d y \leq C_{\alpha} .
$$

Thanks to the definition of $\varphi_{N}(x)$, we can decompose the left side of (2.5) into the following three parts:

$$
\begin{aligned}
& \varphi_{N}(x) \int_{-\infty}^{\infty} e^{-|x-y|} \frac{1}{\varphi_{N}(y)} d y \\
= & \varphi_{N}(x) \int_{-\infty}^{0} e^{-|x-y|} d y+\varphi_{N}(x) \int_{0}^{N} e^{-|x-y|} \frac{1}{(1+y)^{\alpha}} d y \\
& +\frac{\varphi_{N}(x)}{(1+N)^{\alpha}} \int_{N}^{\infty} e^{-|x-y|} d y \\
=: & I+J+K .
\end{aligned}
$$

We estimate $I, J$ and $K$ in the following, respectively.

First, under a simple calculation, it follows that

$$
\int_{-\infty}^{0} e^{-|x-y|} d y= \begin{cases}2-e^{x}, & x \leq 0, \\ e^{-x}, & x>0 .\end{cases}
$$

Hence, when $x \leq 0, I=2-e^{x} \leq 2$. For $x>0$, there exists a constant $C_{\alpha}>0$ (which is independent on $x$ and $N$ ) such that

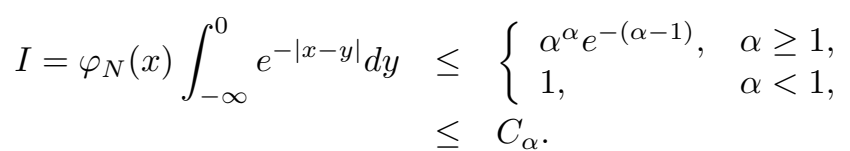


Therefore, there exists a constant $C_{\alpha}$ such that

$$
I \leq C_{\alpha}, \quad \text { for all } x \in \mathbb{R} .
$$

Second, we consider $J$. For $x \leq 0$, one obtains

$$
\begin{aligned}
J & =\int_{0}^{N} e^{x-y} \frac{1}{(1+y)^{\alpha}} d y=e^{x} \int_{0}^{N} \frac{e^{-y}}{(1+y)^{\alpha}} d y \\
& \leq e^{x} \int_{0}^{\infty} e^{-y} d y \leq 1
\end{aligned}
$$

If $x>0$, using the same method as (2.6), we can get

$$
\begin{aligned}
& \int_{0}^{N} e^{-|x-y|} \frac{1}{(1+y)^{\alpha}} d y \leq \int_{\mathbb{R}} e^{-|x-y|} \frac{1}{(1+y)^{\alpha}} d y \\
= & \int_{|y| \leq \frac{|x|}{2}} e^{-|x-y|} \frac{1}{(1+y)^{\alpha}} d y+\int_{|x-y| \leq \frac{|x|}{2}} e^{-|x-y|} \frac{1}{(1+y)^{\alpha}} d y \\
& +\int_{|y| \geq \frac{|x|}{2} \cap|x-y| \geq \frac{|x|}{2}} e^{-|x-y|} \frac{1}{(1+y)^{\alpha}} d y \\
=: & J^{(1)}+J^{(2)}+J^{(3)} .
\end{aligned}
$$

Then when $|y| \leq \frac{|x|}{2}$, one has

$$
J^{(1)} \leq e^{-\frac{|x|}{2}} \int_{|y| \leq \frac{|x|}{2}} \frac{1}{(1+y)^{\alpha}} d y \leq C_{0} e^{-\frac{|x|}{3}} \leq \frac{C_{\alpha}^{(1)}}{(1+x)^{\alpha}},
$$

where the inequality $|x-y| \geq \frac{|x|}{2}$ is used.

Similarly, as $|x-y| \leq \frac{|x|}{2}$, the following estimate for $J^{(2)}$ is true:

$$
J^{(2)} \leq\left(\int_{|x-y| \leq \frac{|x|}{2}} e^{-|x-y|} d y\right) \sup _{|x-y| \leq \frac{|x|}{2}} \frac{1}{(1+y)^{\alpha}} \leq \frac{C_{\alpha}^{(2)}}{(1+x)^{\alpha}}
$$

For $J^{(3)}$, due to the inequality $|x-y| \geq \frac{1}{3}|y|$, it follows that

$$
\begin{aligned}
J^{(3)} & \leq \int_{|y| \geq \frac{|x|}{2}} e^{-\frac{1}{3}|y|} \frac{1}{(1+y)^{\alpha}} d y \leq\left(\int_{\mathbb{R}} e^{-\frac{1}{3}|y|} d y\right) \sup _{|y| \geq \frac{|x|}{2}} \frac{1}{(1+y)^{\alpha}} \\
& \leq \frac{C_{0}}{\left(1+\frac{x}{2}\right)^{\alpha}} \leq \frac{C_{\alpha}^{(3)}}{(1+x)^{\alpha}} .
\end{aligned}
$$

Here we use the fact $\frac{(1+x)^{\alpha}}{\left(1+\frac{x}{2}\right)^{\alpha}} \leq 2^{\alpha}$. Thus, inserting (2.9)-(2.11) into (2.8) and using the definition of $\varphi_{N}(x)$ in (2.1), the inequality

$$
J=\varphi_{N} \int_{0}^{N} e^{-|x-y|} \frac{1}{(1+y)^{\alpha}} d y \leq C_{\alpha} \quad \text { as } x>0
$$

holds true, and here $C_{\alpha}=3 \max \left\{C_{\alpha}^{(1)}, C_{\alpha}^{(2)}, C_{\alpha}^{(3)}\right\}$. Consequently, we can get

$$
J \leq C_{\alpha}, \quad \text { for all } x \in \mathbb{R} \text {. }
$$


Finally, for $K$, we can easily find

$$
\frac{1}{(1+N)^{\alpha}} \int_{N}^{\infty} e^{-|x-y|} d y \leq \begin{cases}1, & x \leq 0 \\ \frac{1}{(1+x)^{\alpha}}, & x \in(0, N) \\ \frac{1}{(1+N)^{\alpha}}, & x \geq N\end{cases}
$$

Thus,

$$
K=\frac{\varphi_{N}}{(1+N)^{\alpha}} \int_{N}^{\infty} e^{-|x-y|} d y \leq C_{\alpha}, \quad \text { for all } x \in \mathbb{R} .
$$

Combining (2.7), (2.12) and (2.13), we obtain inequality (2.5). Therefore we have the estimate

$$
\begin{aligned}
\left|\varphi_{N} \partial_{x} G * f^{2}(x)\right| & =\left|\frac{1}{2} \varphi_{N}(x) \int_{-\infty}^{\infty} e^{-|x-y| / \alpha} f^{2}(y) d y\right| \\
& \leq \frac{1}{2} \varphi_{N}(x) \int_{-\infty}^{\infty} e^{-|x-y|} \frac{1}{\varphi_{N}(y)} \varphi_{N}(y) f(y) f(y) d y \\
& \leq \frac{1}{2}\left(\varphi_{N}(x) \int_{-\infty}^{\infty} e^{-|x-y|} \frac{1}{\varphi_{N}(y)} d y\right)\left\|f \varphi_{N}\right\|_{L^{\infty}}\|f\|_{L^{\infty}} \\
& \leq C_{\alpha}\left\|f \varphi_{N}\right\|_{L^{\infty}}\|f\|_{L^{\infty}}
\end{aligned}
$$

which completes the proof of this lemma.

Remark 2.3. For $\varphi_{N}(x)$ defined in [8, it is obvious that inequality (2.5) holds.

\section{Proof of the MAIN THEOREMS}

Now, we prove Theorem 1.1 first.

Proof. The proof is organized as follows. First, we will give out the estimates on $\|u(x, t)\|_{L^{\infty}}$ and $\left\|u_{x}(x, t)\right\|_{L^{\infty}}$. Then we apply the cut-off function (2.1) to obtain the desired result.

By integration by parts, the Sobolev embedding theorem and Hölder's inequality, we can easily get

$$
\|u(x, t)\|_{L^{\infty}} \leq\left(\left\|u_{0}(x)\right\|_{L^{\infty}}+\int_{0}^{t}\left\|\partial_{x} G *\left(u^{2}+\frac{1}{2} u_{x}^{2}\right)(x, \tau)\right\|_{L^{\infty}} d \tau\right) e^{M t}
$$

and

$$
\left\|u_{x}(x, t)\right\|_{L^{\infty}} \leq\left(\left\|u_{0 x}(x)\right\|_{L^{\infty}}+\int_{0}^{t}\left\|\partial_{x}^{2} G *\left(u^{2}+\frac{1}{2} u_{x}^{2}\right)(x, \tau)\right\|_{L^{\infty}} d \tau\right) e^{2 M t}
$$

where

$$
M=\sup _{t \in[0, T]}\|u(x, t)\|_{H^{s}}, s>\frac{3}{2} .
$$

Next we shall give estimates on $\varphi_{N} u(x, t)$ and $\varphi_{N} u_{x}(x, t)$, where $\varphi_{N}(x)$ is the cut-off function defined as in (2.1). At the same time, we need some tricks to deal 
with the following term as in [16]:

$$
\begin{aligned}
& \int_{-\infty}^{\infty}\left(\varphi_{N}\right)^{2 n-1} u^{2 n-1} \varphi_{N} u_{x} d x \\
= & \int_{-\infty}^{\infty}\left(\varphi_{N} u\right)^{2 n-1} d\left(\varphi_{N} u\right)-\int_{-\infty}^{\infty}\left(\varphi_{N} u\right)^{2 n-1} u\left(\varphi_{N}\right)_{x} d x \\
\leq & \int_{-\infty}^{\infty}\left(\varphi_{N} u\right)^{2 n} d x,
\end{aligned}
$$

where we use the fact (2.2) directly. A similar technique is used to obtain

$$
\int_{-\infty}^{\infty}\left(\varphi_{N}\right)^{2 n-1} u_{x}^{2 n-1} \varphi_{N} u_{x x} d x \leq \int_{-\infty}^{\infty}\left(\varphi_{N} u_{x}\right)^{2 n} d x
$$

Hence, by the same method used in the estimates for (3.1) and (3.2), we get the following inequality:

$$
\begin{aligned}
& \left\|u(x, t) \varphi_{N}\right\|_{L^{\infty}}+\left\|u_{x}(x, t) \varphi_{N}\right\|_{L^{\infty}} \leq e^{2 M t}\left(\left\|u_{0}(x) \varphi_{N}\right\|_{L^{\infty}}+\left\|u_{0 x}(x) \varphi_{N}\right\|_{L^{\infty}}\right) \\
& \quad+e^{2 M t}\left(\int_{0}^{t}\left(\left\|\varphi_{N} \partial_{x} G *\left(u^{2}+\frac{1}{2} u_{x}^{2}\right)\right\|_{L^{\infty}}+\left\|\varphi_{N} \partial_{x}^{2} G *\left(u^{2}+\frac{1}{2} u_{x}^{2}\right)\right\|_{L^{\infty}}\right) d \tau\right) .
\end{aligned}
$$

Therefore, according to (2.3) and (2.4), it follows that there exists a constant $C>0$, which depends on $M>0, T>0$ and $\alpha>1 / 2$, such that

$$
\begin{aligned}
& \left\|u(x, t) \varphi_{N}\right\|_{L^{\infty}}+\left\|u_{x}(x, t) \varphi_{N}\right\|_{L^{\infty}} \\
\leq & C\left(\left\|u_{0}(x) \varphi_{N}\right\|_{L^{\infty}}+\left\|u_{0 x}(x) \varphi_{N}\right\|_{L^{\infty}}\right)+C \int_{0}^{t}\left(\left(\|u(x, \tau)\|_{L^{\infty}}+\left\|u_{x}(x, \tau)\right\|_{L^{\infty}}\right)\right. \\
& \left.\cdot\left(\left\|\varphi_{N} u(x, \tau)\right\|_{L^{\infty}}+\left\|\varphi_{N} u_{x}(x, \tau)\right\|_{L^{\infty}}\right)\right) d \tau \\
\leq & C\left(\left\|u_{0}(x) \varphi_{N}\right\|_{L^{\infty}}+\left\|u_{0 x}(x) \varphi_{N}\right\|_{L^{\infty}}\right. \\
& \left.+\int_{0}^{t}\left(\left\|\varphi_{N} u(x, \tau)\right\|_{L^{\infty}}+\left\|\varphi_{N} u_{x}(x, \tau)\right\|_{L^{\infty}}\right) d \tau\right) .
\end{aligned}
$$

Hence, the following inequality is obtained for any $N \in \mathbb{Z}^{+}$and any $t \in[0, T]$ :

$$
\begin{aligned}
& \left\|u(x, t) \varphi_{N}\right\|_{L^{\infty}}+\left\|u_{x}(x, t) \varphi_{N}\right\|_{L^{\infty}} \\
\leq & C\left(\left\|u_{0}(x) \varphi_{N}\right\|_{L^{\infty}}+\left\|u_{0 x}(x) \varphi_{N}\right\|_{L^{\infty}}\right) \\
\leq & C\left(\left\|u_{0}(x) \max \left(1,(1+x)^{\alpha}\right)\right\|_{L^{\infty}}+\left\|u_{0 x}(x) \max \left(1,(1+x)^{\alpha}\right)\right\|_{L^{\infty}}\right) .
\end{aligned}
$$

Finally, taking the limit as $N$ goes to infinity in the above inequality, we can find that for any $t \in[0, T]$,

$$
\begin{aligned}
& \left(\left|u(x, t)(1+x)^{\alpha}\right|+\left|u_{x}(x, t)(1+x)^{\alpha}\right|\right) \\
\leq & \left(\left\|u_{0}(x) \max \left(1,(1+x)^{\alpha}\right)\right\|_{L^{\infty}}+\left\|u_{0 x}(x) \max \left(1,(1+x)^{\alpha}\right)\right\|_{L^{\infty}}\right) .
\end{aligned}
$$

This completes the proof of Theorem 1.1.

Next, let us write the proof of Theorem 1.2.

Proof. We will use Theorem 1.1 to prove this theorem. For any $t \in[0, T]$, integrating equation (1.2) over the time interval $[0, t]$ we get

$$
u(x, t)-u(x, 0)+\int_{0}^{t} u u_{x}(x, \tau) d \tau+\int_{0}^{t} \partial_{x} G *\left(u^{2}+\frac{1}{2} u_{x}^{2}\right)(x, \tau) d \tau=0 .
$$


By hypothesis and Theorem 1.1 it follows that

$$
\int_{0}^{t_{1}} u u_{x}(x, \tau) d \tau \sim O\left((1+x)^{-\alpha}\right), \quad \text { as } \quad|x| \rightarrow \infty .
$$

At the same time, we have

$$
\begin{aligned}
\int_{0}^{t} \partial_{x} G *\left(u^{2}+\frac{1}{2} u_{x}^{2}\right)(x, \tau) d \tau & =\partial_{x} G * \int_{0}^{t}\left(u^{2}+\frac{1}{2} u_{x}^{2}\right)(x, \tau) d \tau \\
& =\partial_{x} G * \rho(x),
\end{aligned}
$$

while in view of the hypothesis and Theorem 1.1,

$$
0 \leq \rho(x) \sim O\left((1+x)^{-\alpha}\right), \quad \text { as } \quad|x| \rightarrow \infty .
$$

Therefore, using Lemma 2.1, the follow inequality is true:

$$
(1+x)^{\alpha}\left|\partial_{x} G * \rho(x)\right|=\frac{1}{2}(1+x)^{\alpha} \int_{-\infty}^{\infty} e^{-|x-y|} \rho(y) d y \leq C_{\alpha}
$$

Hence, the last term in (3.3) satisfies

$$
\int_{0}^{t} \partial_{x} G *\left(u^{2}+\frac{1}{2} u_{x}^{2}\right)(x, \tau) d \tau \sim O\left((1+x)^{-\alpha}\right), \quad \text { as } \quad|x| \rightarrow \infty .
$$

Thus, we have

$$
u(x, t)-u(x, 0) \sim O\left((1+x)^{-\alpha}\right), \quad \text { as } \quad|x| \rightarrow \infty .
$$

From $u_{0}(x) \sim O\left((1+x)^{-\alpha}\right)$, it is true that $u(x, t) \sim O\left((1+x)^{-\alpha}\right)$, which completes the proof of this theorem.

\section{ACKNOWLEDGMENTS}

The authors would like to thank Lorenzo Brandolese for helpful discussions and the referee for constructive suggestions.

\section{REFERENCES}

1. Bressan and A Constantin, Global conservative solutions of the Camassa-Holm equation. Arch. Ration. Mech. Anal. 183 (2007), no. 2, 215-239. MR.2278406 (2007j:35183)

2. A. Constantin, Finite propagation speed for the Camassa-Holm equation. J. Math. Phys. 46 (2005), no. 2, 023506, 4pp. MR2121730 (2005h:35303)

3. A. Constantin and J. Escher, Well-posedness, global existence and blow-up phenomena for a periodic quasi-linear hyperbolic equation. Comm. Pure Appl. Math. 51 (1998), no. 5, 475-504. MR.1604278 (98k:35165)

4. R. Camassa and D. Holm, An integrable shallow water equation with peaked solitons. Phys. Rev. Lett. 71 (1993), no. 11, 1661-1664. MR1234453 (94f:35121)

5. R. Danchin, A few remarks on the Camassa-Holm equation. Differential Integral Equations 14 (2001), no. 8, 953-988. MR1827098 (2002e:35204)

6. B. Fuchssteiner and A. Fokas, Symplectic structures, their Backlund transformations and hereditary symmetries. Phys. D 4 (1981/82), no. 1, 47-66. MR0636470 (84j:58046)

7. H. P. McKean, Breakdown of a shallow water equation. Mikio Sato: A great Japanese mathematician of the twentieth century. Asian J. Math. 2 (1998), no. 4, 867-874. MR 1734131 (2000k:35262)

8. A. Himonas, G. Misiolek, G. Ponce and Y. Zhou, Persistence properties and unique continuation of solutions of the Camassa-Holm equation. Comm. Math. Phys. 271 (2007), no. 2, 511-522. MR2287915 (2008a:35237)

9. Z. Jiang, L. Ni and Y. Zhou, Wave breaking for the Camassa-Holm equation. Preprint (2010).

10. L. Ni and Y. Zhou, Wave breaking and propagation speed for a class of nonlocal dispersive $\theta$-equations. Nonlinear Anal.: Real World Appl. 12 (2011), no. 1, 592-600. 
11. G. Rodriguez-Blanco, On the Cauchy problem for the Camassa-Holm equation. Nonlinear Anal. 46 (2001), no. 3, Ser. A: Theory Methods, 309-327. MR.1851854(2002i:35172)

12. Z. Xin and P. Zhang, On the weak solution to a shallow water equation. Comm. Pure Appl. Math. 53 (2000), no. 11, 1411-1433. MR1773414 (2001m:35278)

13. Y. Zhou, Wave breaking for a shallow water equation. Nonlinear Anal. 57 (2004), no. 1, 137-152. MR2055991 (2005b:35252)

14. Y. Zhou, Wave breaking for a periodic shallow water equation. J. Math. Anal. Appl. 290 (2004), no. 2, 591-604. MR2033045 (2005c:76021)

15. Y. Zhou, On solutions to the Holm-Staley b-family of equations. Nonlinearity 23 (2010), no. 2, 369-381. MR2578483 (2011a:35483)

16. Y. Zhou and Z. Guo, Blow up and propagation speed of solutions to the DGH equation. Discrete Contin. Dyn. Syst. Ser. B 12 (2009), no. 3, 657-670. MR2525163 (2010m:35475)

Department of Mathematics, Zhejiang Normal University, Jinhua 321004, Zhejiang, People's Republic of China

E-mail address: ni.lidiao@gmail.com

Department of Mathematics, Zhejiang Normal University, Jinhua 321004, Zhejiang, People's Republic of China

E-mail address: yzhoumath@zjnu.edu.cn 